\title{
PROTOCOLO DE CIRURGIA SEGURA: ANÁLISE DA PRODUÇÃO E EXECUÇÃO EM DOIS HOSPITAIS TERCIÁRIOS
}

Surgical safety checklist: analysis of its elaboration and implementation in two tertiary hospitals

Protocolo de cirugía segura: análisis de producción y ejecución en dos hospitales terciarios

Alex Mariano Rosa da Silva ${ }^{1}$ (D), Ivan Tramujas da Costa e Silva² ${ }^{(1)}$, Gisele dos Santos Rocha ${ }^{3}$ (D), Elizabeth Teixeira ${ }^{4}$ (D)

RESUMO: Objetivo: Analisar o processo de produção e execução do protocolo de cirurgia segura em dois hospitais terciários do município de Manaus. Método: Pesquisa guiada pelo design thinking, com ênfase na técnica do duplo diamante, realizada em dois hospitais públicos no município de Manaus, de julho de 2018 a março de 2019. Foi feita nas etapas: investigativa (observação e questionário) e interventiva (síntese, ideação e entrega). Resultados: Na primeira etapa, com 120 horas de observação, constatou-se que as três fases do protocolo não foram cumpridas; analisando-se as repostas a 63 questionários, reforçou-se a não adesão ao checklist. Com base nesses achados, na etapa de síntese, elegeu-se como foco a aplicação do checklist do protocolo; na etapa de ideação, realizou-se a proposição de solução e a testagem-piloto; a etapa de entrega da solução aos hospitais encerrou o ciclo. Conclusão: A análise do processo de execução do protocolo indicou seu descumprimento, o que sugere o comprometimento da segurança do paciente. A solução testada poderá, após entrega e implementação, contribuir para a execução efetiva do protocolo.

Palavras-chave: Segurança do paciente. Centro cirúrgico hospitalar. Enfermagem perioperatória.

ABSTRACT: Objective: To analyze the process of elaboration and implementation of the surgical safety checklist in two tertiary hospitals in the city of Manaus. Method: This study was based on design thinking, focusing on the double diamond technique. It was conducted in two public hospitals in Manaus, from July 2018 to March 2019. The following stages were adopted for this research: investigation (observation and questionnaire) and intervention (synthesis, ideation, and delivery). Results: The first stage, consisting of 120 hours of observation, showed the non-fulfillment of the three phases of the process. After analyzing the answers to the 63 questionnaires, we confirmed the non-adherence to the checklist. Based on these findings, the synthesis phase focused on the use of the checklist; the ideation phase involved the proposal of solutions and the pilot testing; the delivery phase concluded the cycle by providing solutions to the hospitals. Conclusion: The analysis of the process of implementation of the checklist indicated non-compliance, suggesting risk to patient safety. After delivery and implementation, the tested solution may contribute to the effective execution of the checklist. Keywords: Patient safety. Surgery department, hospital. Perioperative nursing.

RESUMEN: Objetivo: Analizar el proceso de producción y ejecución del Protocolo de Cirugía Segura en dos hospitales terciarios de la ciudad de Manaus. Método: Investigación guiada por Design Thinking, con énfasis en la técnica Double Diamond, llevada a cabo en dos hospitales públicos de la ciudad de Manaus, desde julio de 2018 hasta marzo de 2019. Se realizó por etapas: de investigación (observación y cuestionario) e intervencionista (síntesis, ideación y entrega). Resultados: En la primera etapa, con 120 horas de observación, se encontró que las tres fases del protocolo no se cumplieron; En base a las respuestas a 63 cuestionarios, se reforzó la no adhesión a la lista de verificación. Con base en estos hallazgos, en la etapa de síntesis, la aplicación de la lista de verificación del protocolo fue elegida como el foco; en la etapa de ideación, se llevaron a cabo la propuesta de solución y la prueba piloto;

'Mestre em Cirurgia pela Universidade Federal do Amazonas (UFAM). Acadêmico medicina na Universidade Nilton Lins - Manaus (AM), Brasil.

${ }^{2}$ Doutor em Medicina Tropical pela Universidade do Estado do Amazonas (UEA). Docente permanente do Programa de Pós-Graduação em Cirurgia da Faculdade de Medicina da UFAM - Manaus (AM), Brasil. ${ }^{3}$ Mestre em Enfermagem pela UFAM. Docente do curso de Graduação em Enfermagem da UEA - Manaus (AM), Brasil.

${ }^{4}$ Doutora em Ciências Socioambientais pela Universidade Federal do Pará. Docente permanente do Mestrado Profissional de Enfermagem em Saúde Pública da UEA - Manaus (AM), Brasil.

*Autora correspondente: grocha@uea.edu.br

Recebido: 16/04/2019 - Aprovado: 22/07/2020

https://doi.org/10.5327/Z1414-4425202000030002 
La etapa de entrega de la solución a los hospitales finalizó el ciclo. Conclusión: El análisis del proceso de ejecución del protocolo indicó incumplimiento, lo que sugiere comprometer la seguridad del paciente. La solución probada puede, después de la entrega y la implementación, contribuir a la ejecución efectiva del protocolo.

Palabras clave: Seguridad del paciente. Servicio de cirugía en hospital. Enfermería perioperatoria.

\section{INTRODUÇÃO}

Mundialmente, a preocupação com a segurança dos pacientes nas instituições de saúde vem progredindo. As pesquisas nessa área se iniciaram em 1974 e alcançaram o grande marco no ano de 1999, após a divulgação do relatório do Institute of Medicine To Err is Human, com o resultado de estudo realizado em hospitais dos Estados Unidos da América (EUA), em que foi constatado que cerca de 100 mil pessoas morriam vítimas de eventos adversos, ou seja, danos causados durante os cuidados à saúde e que não têm relação com a doença do paciente ${ }^{1}$.

No Brasil, a motivação gerada em âmbito internacional para a mobilização em prol da segurança do paciente teve início em 2001, quando foi criado o Projeto Hospitais Sentinela com o intuito de ampliar e sistematizar a vigilância de produtos utilizados em serviços de saúde, para garantir mais segurança e qualidade a pacientes e profissionais ${ }^{2}$. Em 2008, foi criada, pela Organização Pan-Americana de Saúde (OPAS), a Rede Brasileira de Enfermagem e Segurança do Paciente (REBRAENSP), que tem como objetivo disseminar a cultura de segurança do paciente para as instituições, trabalhadores e familiares de pacientes ${ }^{3}$.

A Organização Mundial da Saúde (OMS), com o auxílio de colaboradores de diversos países, desenvolveu uma lista de verificação cirúrgica (checklist) a ser usada no período transoperatório, guiada por três princípios: simplicidade, ampla aplicabilidade e possibilidade de mensuração do impacto. Permite, assim, que as equipes sigam de maneira eficiente as etapas críticas de segurança e minimizem os riscos evitáveis mais comuns, que colocam em perigo as vidas e o bem-estar dos pacientes ${ }^{3,4}$.

Em 2009 foi publicado, em português, pelo Ministério da Saúde juntamente com a OPAS, o manual de implementação de medidas para o projeto de Segurança do Paciente Cirurgias Seguras Salvam Vidas. Em estudo realizado nos anos de 2009 e 2010, após a implantação da cirurgia segura, houve redução de $36 \%$ das complicações e de $47 \%$ da mortalidade em pacientes cirúrgicos ${ }^{4}$. A segurança do paciente compõe um eixo de atenção que se compromete a oferecer cuidado livre de danos e lesões acidentais durante a prestação de assistência à saúde ${ }^{4}$.

O Protocolo de Cirurgia Segura (PCS), criado pela OMS, vem sendo instituído nos hospitais brasileiros no intuito de garantir a segurança do paciente cirúrgico ${ }^{4,5}$. É considerada uma ferramenta que auxilia na promoção do trabalho em equipe daqueles que atuam no ato anestésico-cirúrgico, promovendo a segurança do paciente, além de favorecer a capacitação dos profissionais, a melhor compreensão das ações necessárias para o fortalecimento dos processos de segurança do paciente, ou seja, a percepção do risco como forma efetiva para a mudança prática de instituir as medidas preventivas ${ }^{5,6}$.

O enfermeiro que atua em contexto cirúrgico pode identificar problemas e incentivar a produção de dispositivos e soluções tecnológicas?. Os possíveis erros, as dificuldades e as fragilidades que podem colocar em risco a segurança do paciente cirúrgico devem ser solucionados pela aplicação adequada e plena de dispositivos que proporcionem estratégias para uma assistência segura e de qualidade ${ }^{7}$.

Com base nisso, delineou-se a questão de pesquisa: como se desenvolve o processo de execução do PCS em dois hospitais gerais de grande porte do município de Manaus?

\section{OBJETIVO}

Analisar o processo de produção e execução do PCS em dois hospitais do município de Manaus.

\section{MÉTODO}

Pesquisa do tipo metodológica ${ }^{8}$, guiada pelos passos do design thinking, com ênfase na técnica do duplo diamante ${ }^{9,10}$. $\mathrm{Na}$ área da saúde, o método design thinking vem sendo utilizado com sucesso e está contribuindo para a resolução de problemas em diversos temas, como a humanização de serviços, a atenção às necessidades dos usuários e a melhoria do fluxo perioperatório ${ }^{10}$. 
Um modelo proposto para a execução do design thinking é a técnica do duplo diamante (do inglês double diamond), criada em 2004 pelo British Design Council ${ }^{11}$. Para o desenvolvimento da técnica do duplo diamante, é necessário cumprir quatro etapas: descoberta (pesquisa), que busca conhecer o problema que se pretende resolver; definição (síntese), que é a identificação da área a ser enfocada; desenvolvimento (ideação), em que soluções potenciais para o problema são construídas e testadas; implementação (entrega), que é a oferta das soluções que melhor funcionem ${ }^{10,11}$.

Desse modo, esta pesquisa adaptou a condução do duplo diamante, que foi realizada nas seguintes etapas: etapa investigativa (descoberta - realização de observação e aplicação de questionários), que ocorreu entre julho e setembro de 2018; etapa interventiva (síntese, ideação com testagem piloto e entrega), entre outubro de 2018 e março de 2019. As duas fases foram realizadas em dois centros cirúrgicos de dois hospitais públicos de grande porte do município de Manaus, Amazonas, Brasil, sendo um estadual, referência em cirurgias ortopédicas (Hospital A) e um federal, universitário (Hospital B).

Participaram 63 profissionais (14 enfermeiros, 15 médicos-cirurgiões, 13 médicos anestesiologistas, 14 técnicos de enfermagem e sete instrumentadores cirúrgicos). A amostra foi definida por conveniência. Os critérios de inclusão foram: atuar nos dois centros cirúrgicos observados e ter, no mínimo, um ano de experiência com atividades cirúrgicas.

Utilizou-se para a coleta de dados a observação não intervencionista (shadowing), apoiada pelo PCS e um questionário adaptado $^{11}$. A observação nos dois hospitais foi realizada pelo primeiro pesquisador, após aceite e consentimento da equipe cirúrgica, e ocorreu de forma concomitante nos dois hospitais (Hospital A pela manhã e Hospital B à tarde). Durante os intervalos da observação (período entre uma cirurgia e o início da seguinte), o pesquisador conversava com os profissionais sobre o objetivo da pesquisa e oferecia o questionário para ser preenchido, o qual visava verificar o conhecimento, as facilidades, as dificuldades e as sugestões referidas pelos profissionais para a realização do PCS. O preenchimento do questionário pela equipe cirúrgica ocorreu nas dependências do centro cirúrgico (CC), em horários previamente acertados com os participantes de acordo com a disponibilidade manifestada, para posterior devolução ao pesquisador.

Os dados foram analisados por meio de estatística descritiva quantitativa, segundo média e porcentagem. Foi realizada a distribuição de frequência absoluta (n) e relativa (\%) dos dados enumerados (atributos ou dados nominais) e a estatística descritiva dos dados quantitativos (grandezas específicas ou variáveis). Os dados foram organizados no programa Microsoft ${ }^{\circledast}$ Excel 2013 e analisados pelo programa estatístico Statistical Package for the Social Sciences (SPSS) versão 21.

Os aspectos éticos foram respeitados em todas as etapas do estudo, em concordância com a Resolução n ${ }^{\circ}$ 466/2012, do Conselho Nacional de Saúde. Foi obtida aprovação da pesquisa pelo Comitê de Ética em Pesquisa da Universidade Federal do Amazonas (CEP/UFAM) e o projeto foi aprovado pelo Certificado de Apresentação para Apreciação Ética (CAAE) $n^{\circ}$ 92500817.9.0000.5020. Este artigo faz parte da dissertação de mestrado intitulada Programa de cirurgia segura: proposta para consolidar a implementação em dois hospitais públicos terciários em Manaus.

\section{RESULTADOS}

Quanto à etapa investigativa, foram realizadas 120 horas de observação, 60 em cada hospital, nos respectivos blocos cirúrgicos (BC). A média diária de cirurgias observadas em ambos os hospitais foi de 20 procedimentos, totalizando 200 cirurgias acompanhadas em 10 dias em cada hospital. Tais observações concentraram-se, principalmente, no início e no término das cirurgias.

Em relação ao momento antes da indução anestésica (sign in), verificou-se que em 197 procedimentos (98,5\%), em ambos os hospitais, não foi realizada a demarcação do sítio cirúrgico conforme é recomendado no PCS, salvo em pacientes de cirurgia plástica. Em três procedimentos (1,5\%) desse último tipo, os pacientes foram examinados pelo cirurgião ou médico residente para constatar o lado da cirurgia e realizar a demarcação. Nesses três casos, foi constatado que a demarcação do sítio cirúrgico não foi empregada de forma correta. Em relação ao momento antes da incisão cirúrgica (timeout), nos dois hospitais não se observou a presença do enfermeiro no momento da realização da pausa de segurança. Percebeu-se que, em ambos os hospitais, em nenhum procedimento houve a execução do timeout nem a confirmação da identificação da equipe cirúrgica com a verbalização, por parte de cada membro, do seu nome e função antes da incisão cirúrgica, conforme recomenda o PCS.

Durante a observação do timeout, os resultados permitiram identificar itens que não foram executados conforme o estabelecido pelo PCS, como:

- ausência do enfermeiro no momento da realização do timeout, já que ele estava ocupado com outras atividades administrativas; 
- circulante que ficava impedido de realizar a declamação da lista de verificação porque estava executando várias tarefas na sala operatória $(\mathrm{SO})$;

- falta de declamação da lista de verificação em razão da ausência de um profissional com atribuição específica para a sua execução.

Em relação ao momento antes de o paciente sair da $\mathrm{SO}$ (sign out) em ambos os hospitais, em 150 procedimentos (75\%) foi realizada a contagem de instrumentos, de agulhas e de compressas. Não se verificou em nenhuma cirurgia, durante o período de observação, o registro sobre se os equipamentos funcionaram adequadamente durante os procedimentos. Sobre esse aspecto, notou-se que em 75 procedimentos $(37,5 \%)$ ocorreram falhas técnicas na monitorização e nas leituras dos parâmetros, como oximetria de pulso, frequência cardíaca e pressão arterial não invasiva, e os equipamentos tiveram de ser substituídos. Viu-se que equipamentos como monitores e carro de anestesia não passavam por testes antes dos procedimentos cirúrgicos e, ainda, estavam com a calibração vencida ou não apresentavam identificação da última calibração ou checagem pela engenharia clínica.

A segunda parte da etapa investigativa contou com a aplicação dos questionários, que se iniciavam com os dados do perfil dos entrevistados (Tabela 1). Quanto aos profissionais, 30 participantes eram do Hospital A e 33 do Hospital B.

Em relação à segunda parte do questionário, a Tabela 2 mostra a porcentagem das respostas das questões de 1 a 3, com dados totais dos dois hospitais. Salienta-se que o número de participantes é divergente na segunda e na terceira perguntas, pois três dos 63 profissionais que compuseram a amostra referiram não conhecer o PCS.

Sobre a aplicação do PCS, foi solicitado que os profissionais, no fim do questionário, apontassem as principais facilidades e dificuldades para a sua execução. As respostas coletadas nos dois hospitais pesquisados são mostradas na Tabela 3.

As etapas interventivas (síntese, ideação com testagem piloto e entrega) ocorreram após o término da análise da etapa anterior (etapa investigativa). Na etapa de síntese foi redigido um relatório e elegeu-se como aspecto a ser enfocado nas etapas subsequentes o checklist. A escolha do foco consubstancia-se nos resultados da etapa anterior, que apontaram, entre outros aspectos, que o checklist ou já chegava preenchido no prontuário do paciente, com as etapas

Tabela 2. Respostas do questionário sobre o PCS pelos profissionais dos hospitais.

\begin{tabular}{|c|c|c|}
\hline Questões & $\begin{array}{l}\text { Sim } \\
\text { n (\%) }\end{array}$ & $\begin{array}{l}\text { Não } \\
\text { n (\%) }\end{array}$ \\
\hline 1. Você conhece o PCS da OMS? $(n=63)$ & $60(95,2)$ & $3(4,7)$ \\
\hline $\begin{array}{l}\text { 2. O PCS é aplicado em todas as } \\
\text { cirurgias no hospital? }(n=60)\end{array}$ & $27(45,0)$ & $33(55,0)$ \\
\hline $\begin{array}{l}\text { 3. Você utiliza a lista de } \\
\text { verificação do PCS? }(n=60)\end{array}$ & $35(58,3)$ & $25(41,6)$ \\
\hline
\end{tabular}

PCS: protocolo de cirurgia segura; OMS: Organização Mundial da Saúde.

Tabela 1. Perfil dos profissionais participantes da pesquisa $(n=63)$.

\begin{tabular}{|c|c|c|c|c|c|}
\hline \multirow{2}{*}{ Variável } & \multicolumn{5}{|c|}{ Profissão } \\
\hline & Enfermeiro & Cirurgião & Anestesiologista & Técnico de Enfermagem & Instrumentador \\
\hline Idade média (anos) & 34,7 & 35,5 & 42,3 & 39,4 & 29,3 \\
\hline \multirow[t]{2}{*}{$\begin{array}{l}\text { Tempo médio de } \\
\text { atuação (anos) }\end{array}$} & 6,7 & 5,5 & 11,7 & 10,8 & 3,5 \\
\hline & $n(\%)$ & $n(\%)$ & $n(\%)$ & n (\%) & $n(\%)$ \\
\hline \multicolumn{6}{|l|}{ Sexo biológico } \\
\hline Masculino & $6(43,7)$ & $12(80,0)$ & $7(53,3)$ & $4(37,5)$ & $2(33,3)$ \\
\hline Feminino & $8(56,3)$ & $3(20,0)$ & $6(46,7)$ & $10(62,5)$ & $5(66,7)$ \\
\hline \multicolumn{6}{|l|}{ Titulação } \\
\hline Especialista & $14(87,5)$ & $14(93,3)$ & $13(86,6)$ & $0(0,0)$ & $0(0,0)$ \\
\hline Médio & $0(0,0)$ & $0(0,0)$ & $0(0,0)$ & $0(0,0)$ & $7(100)$ \\
\hline Mestrado & $2(12,5)$ & $1(6,7)$ & $2(13,4)$ & $0(0,0)$ & $0(0,0)$ \\
\hline Residência & $0(0,0)$ & $0(0,0)$ & $0(0,0)$ & $0(0,0)$ & $0(0,0)$ \\
\hline Técnico & $0(0,0)$ & $0(0,0)$ & $0(0,0)$ & $14(100)$ & $0(00,0)$ \\
\hline
\end{tabular}


Tabela 3. Facilidades e dificuldades apontadas pelos profissionais na implantação do PCS ( $n=60)$.

\begin{tabular}{|l|l|l|}
\hline & \multicolumn{2}{|c|}{ Respostas } \\
\cline { 2 - 3 } & $\mathbf{n}$ & $\%$ \\
\hline Facilidades & & \\
\hline Torna o procedimento mais seguro & 31 & 51,6 \\
\hline Promove melhoria do serviço & 21 & 35,0 \\
\hline Promove redução de erros médicos & 30 & 50,0 \\
\hline Torna a comunicação mais eficaz & 21 & 35,0 \\
\hline Incorre em benefícios para a instituição & 23 & 38,3 \\
\hline $\begin{array}{l}\text { Evita o uso de equipamento defeituoso } \\
\text { Dificuldades }\end{array}$ & 15 & 25,0 \\
\hline É mais um papel burocrático & & \\
\hline $\begin{array}{l}\text { Há demora e atraso no serviço ao } \\
\text { usar o timeout }\end{array}$ & 17 & 28,3 \\
\hline $\begin{array}{l}\text { Falta de conhecimento do PCS pela equipe } \\
\text { de saúde }\end{array}$ & 39 & 65,0 \\
\hline PCS: protocolo de cirurgia segura. & 13 & 21,6 \\
\hline
\end{tabular}

carimbadas, ou era preenchido no fim do ato anestésico-cirúrgico, quando cada profissional realizava sua parte no preenchimento e o carimbava. Isso se dava em descumprimento ao recomendado pelo protocolo da OMS, segundo o qual o PCS deve ser realizado por um só profissional.

Os resultados também apontaram, com ênfase, a resistência de toda a equipe envolvida na cirurgia para a realização do checklist, principalmente quando os procedimentos cirúrgicos aconteceram seguidamente, um após o outro. Ademais, em todos os processos observados, houve também resistência entre os cirurgiões na apresentação verbal da equipe. Quanto à existência de norma, observou-se não haver normativa específica estabelecida para o cumprimento do checklist em ambos os hospitais.

$\mathrm{Na}$ etapa de ideação, procedeu-se à proposição de uma solução para a execução plena do PCS e a testagem-piloto. Elaborou-se um modelo com cinco etapas, denominado de Modelo de Cinco Pontos, assim constituído:

- $1^{\circ}$ ponto: treinamento e sensibilização da equipe cirúrgica;

- $2^{\circ}$ ponto: normatização e nomeação do líder para a execução do PCS;

- $3^{\circ}$ ponto: criação do dia " $D$ " para a execução do PCS;

- $4^{\circ}$ ponto: supervisão do cumprimento das normas;

- $5^{\circ}$ ponto: divulgação das estatísticas com os resultados (Figura 1).

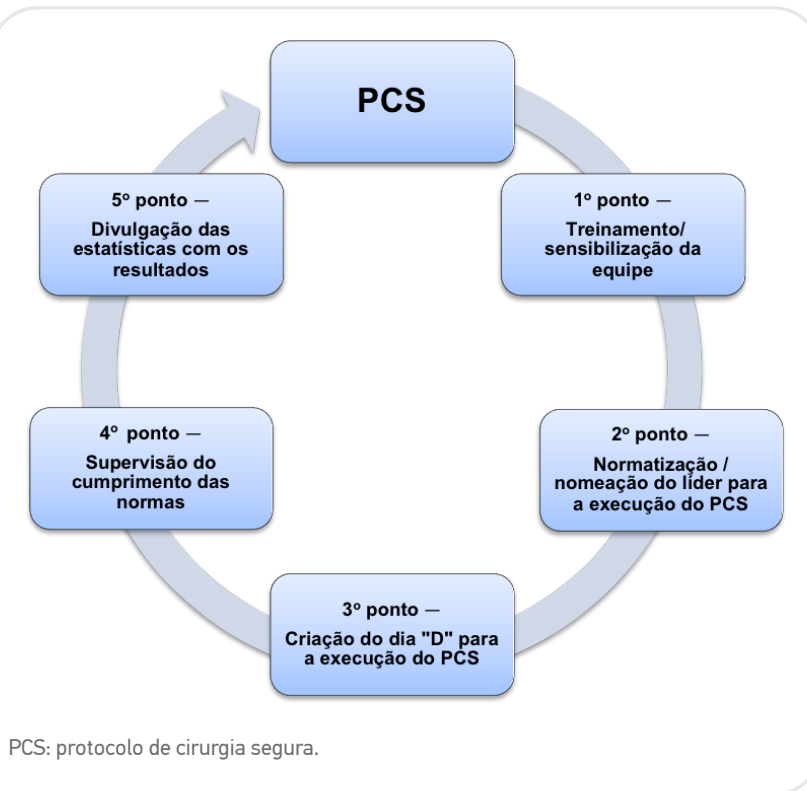

Figura 1. Modelo de cinco pontos proposto para a efetivação da execução do PCS.

Com vistas a obter apoio para a testagem piloto do modelo, no mês de janeiro de 2019 se realizaram reuniões das quais participaram, nos dois hospitais: diretor-geral, diretor clínico, diretor de ensino e pesquisa, coordenação de residência médica e de enfermagem, coordenação do núcleo de segurança do paciente, gerente de enfermagem e do BC. Acordou-se adesão plena ao modelo e sua respectiva testagem piloto.

Em seguida, nos meses de fevereiro e março de 2019, testou-se o modelo nos dois BC. Ao término, verificou-se, com nova observação diária nos dois hospitais (pelo período de 10 dias) e coletando as opiniões dos gestores hospitalares por meio de diálogo, que houve maior sensibilização por parte da equipe cirúrgica e a realização do PCS com mais efetividade. No entanto, em alguns procedimentos, continuou não havendo a declamação do protocolo e havendo resistências por parte dos profissionais envolvidos, o que implica a necessidade de intervenção em médio prazo, direta e contínua, por parte dos gestores hospitalares.

\section{DISCUSSÃO}

Os resultados da primeira etapa (investigativa), em seu período de observação, com a verificação do estado atual da prática nos dois hospitais investigados quanto aos procedimentos que devem ser feitos antes da indução anestésica, como a 
demarcação do sítio cirúrgico, mostraram que eles não foram executados de forma correta em nenhum dos dois hospitais. A identificação/demarcação do sítio cirúrgico, conforme o protocolo de segurança do paciente, deve ser realizada pelo cirurgião responsável pelo procedimento cirúrgico antes do encaminhamento do paciente para a SO. É indicado que o paciente esteja acordado e consciente, se possível, para que ele possa confirmar o local do procedimento. O local, no corpo do paciente, que indica onde ele será operado deve ser marcado com caneta dermográfica. "A instituição deverá ter processos definidos por escrito para lidar com as exceções, como, por exemplo, recusa documentada do paciente, de modo a garantir a segurança cirúrgica" ${ }^{\prime 2}$.

Estudo aponta que, nos EUA, estimou-se que cirurgias em local errado e no paciente errado ocorreram em cerca de um em cada 50 mil procedimentos, o que equivale a 1.500-2.500 incidentes por ano. Após a implementação do checklist do PCS nas unidades hospitalares, "as mortes provocadas por complicações cirúrgicas em local errado e no paciente errado foram reduzidas em quase a metade (de $1,5 \%$ a $0,8 \%)^{\prime \prime 13}$.

Em relação ao timeout, ou seja, momentos antes da incisão cirúrgica ou intervalo de confiança, houve falta de declamação da lista de verificação por causa da ausência de um profissional com atribuição específica para sua execução. A mesma situação foi relatada em estudos realizados em um hospital universitário e em um hospital geral, onde, na etapa do timeout, em nenhuma das cirurgias se constatou a apresentação da equipe cirúrgica antes do procedimento cirúrgico, a identificação do paciente e a marcação do local de cirurgia ${ }^{13,14}$.

Ao término do procedimento, antes de o paciente sair da sign out (SO), deve-se realizar a conferência dos instrumentais, contar compressas e agulhas, identificar o espécime para estudo anatomopatológico e reportar se há algum problema com equipamentos para ser resolvido ${ }^{4}$. A observação nos dois hospitais identificou que a contagem dos instrumentais não foi realizada em nenhuma das cirurgias, mesmo diante da presença do instrumentador cirúrgico.

Em estudo realizado sobre a retenção inadvertida de objetos intracavitários, $90 \%$ eram têxteis, 5,21\% instrumentais cirúrgicos e 2,84\% agulhas. Entre os motivos relatados para tal ocorrência, a ausência de contagem esteve associada a $25 \%$ dos casos. A medida preventiva mais propalada é a contagem desses materiais de forma rotineira, o que não é padronizado em muitos $\mathrm{CC}^{15}$.

A observação permitiu refletir sobre a resistência dos profissionais à implementação do PCS como rotina. Estudos apontam que a realização desse protocolo vem sendo estimulada no Brasil. Porém, rotinas novas nem sempre são recebidas positivamente num primeiro momento, causando resistência por parte de membros da equipe cirúrgica, com destaque para os médicos-cirurgiões e anestesiologistas, principalmente quando a eficácia de seus resultados, embora decisiva, não é facilmente demonstrada em curto prazo ${ }^{16,17}$.

No que se refere às entrevistas realizadas nos dois hospitais pesquisados, que abrangeram os $63(100 \%)$ profissionais envolvidos, constatou-se que a maioria deles conhecia a existência do PCS e somente um número mínimo, de três (7\%), a desconhecia. Considerando-se que o PCS foi implantado em média há dois anos em ambos os hospitais, seria de se esperar que todos os profissionais o conhecessem.

Esse achado corrobora um estudo que identificou que muitos cirurgiões e médicos residentes em cirurgia não conheciam o checklist proposto pela OMS, e outros médicos, apesar de já o possuírem até como aplicativo pessoal, não o utilizavam ${ }^{18}$. O trabalho ainda apontou que, da equipe de enfermagem, poucos membros não conheciam o checklist de cirurgia segura ${ }^{18}$.

Entre as facilidades da implantação do PCS reconhecidas e apontadas pelos profissionais neste estudo, o fato de tornar o procedimento seguro foi a mais apontada, seguida da redução dos erros médicos e do fato de propiciar benefício para a instituição. Diante disso, nota-se que os profissionais envolvidos demonstraram, em tese, confiança no protocolo, pois reconheceram que seu uso pode levar ao decréscimo de eventos adversos, trazendo mais segurança ao paciente cirúrgico.

Com relação às dificuldades elencadas pelos profissionais para a implantação do PCS, a opção mais indicada foi a de ele constituir-se em mais um papel burocrático a ser exercido, mas isso também porque o timeout demora muito e atrasa o serviço. Ainda, foi mencionada a falta de conhecimento sobre o programa pela equipe. Tal relato confirma o que foi observado quanto à resistência do público-alvo entrevistado à declamação do checklist, desconsiderando seu papel como ferramenta criada para assegurar a diminuição dos riscos inerentes à realização de procedimentos anestésico-cirúrgicos, porém há o reconhecimento da necessidade de maior conhecimento por parte da equipe sobre o PCS.

Em estudo sobre a adesão ao checklist do PCS, identificou-se em 32 publicações sobre o tema que "a viabilidade da lista de verificação de segurança cirúrgica vem se apresentando promissora em diversos hospitais brasileiros, embora haja, ainda, baixo engajamento da equipe cirúrgica em sua adesão"19. A pesquisa aponta ser necessário um coordenador para viabilizar a verificação da lista e sugere o enfermeiro 
como coordenador do BC, pois ele pode utilizar essa ferramenta como forma de mensurar e avaliar a assistência prestada ao paciente cirúrgico ${ }^{19}$.

Em investigação realizada nos EUA sobre a implementação do checklist em hospitais, concluiu-se que o sucesso no processo de execução do PCS está associado à melhor percepção dos profissionais quanto ao seu uso, ao respeito mútuo entre a equipe cirúrgica, à liderança, à coordenação e à comunicação da equipe. Esse estudo comprovou, ainda, que após a implantação do PCS a melhora na segurança perioperatória foi percebida pelos profissionais envolvidos ${ }^{20}$.

O processo de implementação do PCS aparentemente é simples, mas acaba tornando-se um processo complexo por necessitar do enfrentamento da resistência à mudança pelos profissionais envolvidos, que terão suas rotinas no ambiente de trabalho alteradas, tanto mais quanto maior for a complexidade do local em que o protocolo deve ser implantado ${ }^{18-20}$.

Quanto à etapa interventiva, após a síntese da etapa anterior, percebeu-se a necessidade de implantar um modelo inovador para o aperfeiçoamento da execução do PCS, identificada diante de alguns conflitos, principalmente a resistência da não aceitação e a falta de execução do protocolo na íntegra nos dois hospitais campos da pesquisa. $\mathrm{O}$ intuito foi contribuir com algumas intervenções que pudessem diminuir tais conflitos. Assim procedendo, além de aplicar o modelo proposto conseguiu-se estabelecer novos recursos práticos para a melhoria da efetividade do PCS, pois, nos dois hospitais, constatou-se que não havia ferramentas que auxiliassem na implantação e na execução do programa. Com a operacionalização do modelo, espera-se que haja melhor adesão à execução do PCS, aumentando a qualidade da assistência prestada ao paciente cirúrgico, colaborando para diminuir eventuais eventos adversos e, consequentemente, reduzindo custos hospitalares.

Estudos evidenciam a importância do papel do enfermeiro na execução do PCS, seja pela sua capacidade de gerenciamento da equipe multiprofissional, seja pela sua riqueza de conhecimento, que pode levar benefícios aos profissionais de saúde e pacientes, proporcionando o aumento da segurança cirúrgica ${ }^{5-7}$.

Quanto à limitação do estudo, pode-se considerar o fato de ter sido realizado em apenas dois dos hospitais da rede de atenção da cidade de Manaus. Outra limitação foi a não validação do modelo proposto na pesquisa por juízes especialistas da área, o que será realizado em trabalho posterior.

\section{CONCLUSÃO}

A análise do processo de construção e execução do PCS indicou o descumprimento das fases do referido protocolo, o que sugere comprometimento da segurança do paciente. A atuação do enfermeiro, no contexto cirúrgico, consiste em identificar problemas e incentivar a proposição de soluções para uma assistência de qualidade e livre de riscos. Os resultados indicam a necessidade de soluções intra-hospitalares, envolvendo todos os profissionais para aumentar a adesão ao PCS. Sendo assim, acredita-se que o modelo proposto e testado durante este estudo, após sua implementação nos dois hospitais, poderá contribuir para a execução com maior efetividade do PCS.

\section{REFERÊNCIAS}

1. Brasil. Ministério da Saúde. Documento de referência para o Programa Nacional de Segurança do Paciente [Internet]. Brasília: Ministério da Saúde; 2014 [acessado em 3 maio 2019]. Disponível em: http://bvsms. saude.gov.br/bvs/publicacoes/documento_referencia_programa_ nacional_seguranca.pdf

2. Elias ACGP, Schimidt DRC, Yonekura CS, Dias A O, Ursi ES, Silva RPJ, et al. Avaliação da adesão ao checklist de cirurgia segura em hospital universitário público. Rev SOBECC [Internet]. 2015 [acessado em 3 maio 2019];20(3):128-33. Disponível em: https://doi.org/10.5327/ Z1414-4425201500030002

3. Nascimento JC, Draganov PB. História da qualidade em segurança do paciente. Hist Enferm Rev Eletrônica [Internet]. 2015 [acessado em
7 maio 2019];6(2):299-309. Disponível em: http://here.abennacional. org.br/here/seguranca_do_paciente.pdf

4. Brasil. Ministério da Saúde. Portaria nº 2.095, de 24 de setembro de 2013. Institui os Protocolos Básicos de Segurança do Paciente [Internet]. Brasília: MS; 2013 [acessado em 3 maio 2019]. Disponível em: https://bvsms.saude.gov.br/bvs/saudelegis/gm/2013/ prt2095_24_09_2013.html

5. Diego LAS, Salman FC, Silva JH, Brandão JC, Oliveira Filho G, Carneiro $A F$, et al. Construction of a tool to measure perceptions about the use of the World Health Organization Safe Surgery Checklist Program. Braz J Anestesiol [Internet]. 2016 [acessado em 2 maio 2019];66(4):351-5. Disponivel em: https://doi.org/10.1016/j.bjane.2014.11.011 
6. Mafra CR, Rodrigues MCS. Lista de verificação de segurança cirúrgica: uma revisão integrativa sobre benefícios e sua importância. Rev Fund Care Online [Internet]. 2018 [acessado em 3 maio 2019];10(1):268-75. Disponível em: https://doi.org/10.9789/2175-5361.2018.v10i1.268-275

7. Vasconcelos MGV, Migoto MT, Silva AC. 0 enfermeiro na execução do checklist em centro cirúrgico: uma revisão integrativa. RGS [Internet]. 2018 [acessado em 3 maio 2019];19(1):57-68. Disponível em: http://www.herrero.com.br/files/revista/ file7e65885e60831dd68cb383fac0e158b0.pdf

8. Caldas ACS, Dias RS, Sousa SMA, Teixeira E. Produção sensível e criativa de tecnologia cuidativo-educacional para famílias de crianças com gastrostomia. Esc Anna Nery. 2019;23(1):e20180144. http:// dx.doi.org/10.1590/2177-9465-ean-2018-0144

9. Brown T. Design Thinking: uma ferramenta metodológica poderosa para decretar o fim das velhas ideias. São Paulo: Alta Books; 2017.

10. Caulliraux AA. Proposta de método para aplicação do design thinking em serviços de saúde: o caso do fluxo perioperatório do paciente eletivo [dissertação]. Rio de Janeiro: Universidade Federal Fluminense; 2015

11. Design Council. Design for Public good [Internet]. Londres: Design Council; 2013 [acessado em 3 maio 2019]. Disponível em: https:// www.tdm-recruitment.com

12. Ribeiro HCTC, Quites HFO, Bredes AC, Sousa KAS, Alves M. Adesão ao preenchimento de segurança cirúrgica. Cad Saúde Pública [Internet]. 2017 [acessado em 3 maio 2019];33(10):e00046216. Disponível em: http://dx.doi.org/10.1590/0102-311x00046216

13. Oliveira AC, Abreu AR, Almeida SSA. Implementação do checklist de cirurgia segura em um hospital universitário. Enferm Foco [Internet]. 2017 [acessado em 3 maio 2019];8(4):14-8. Disponível em: http:// revista.cofen.gov.br/index.php/enfermagem/article/view/972 https://doi.org/10.21675/2357-707X.2017.v8.n4.972

14. Martins GS, Carvalho R. Realização do timeout pela equipe cirúrgica: facilidades e dificuldades. Rev SOBECC [Internet]. 2014 [acessado em 3 maio 2019];19(1):18-25. Disponível em: http:// www.sobecc.org.br/arquivos/artigos/2015/pdfs/site_sobecc_ v19n1/04_sobecc v19n1.pdf

http://dx.doi.org/10.4322/sobecc.2014.007

15. Birolini DV, Rasslan S, Utiyama EM. Retenção inadvertida de corpos estranhos após intervenções cirúrgicas: análise de 4547 casos. Rev Col Bras Cir [Internet]. 2016 [acessado em 3 maio 2019];43(1):12-7. Disponível em: http://www.scielo.br/pdf/rcbc/v43n1/pt_0100-6991rcbc-43-01-00012.pdf http://dx.doi.org/10.1590/0100-69912016001004

16. Pancieri AP, Carvalho R, Braga EM. Aplicação do checklist para cirurgia segura: relato de experiência. Rev SOBECC [Internet]. 2014 [acessado em 3 maio 2019]; 19(1):26-33. Disponível em: http://www. sobecc.org.br/arquivos/artigos/2015/pdfs/site_sobecc_v19n1/05_ sobecc_v19n1.pdf http://dx.doi.org/10.4322/sobecc.2014.006

17. Cabral RA, Eggenberger TK, Keller K, Gallison BS, Newman D. Use of a Surgical Safety Checklist to improve team communication. AORN J [Internet]. 2016 [acessado em 3 maio 2019];104(3):206-16. Disponível em: https://www.ncbi.nlm.nih.gov/pubmed/27568533 https://doi.org/10.1016/j.aorn.2016.06.019

18. Molina G, Jiang W, Edmondson L, Gibbons L, Huang LC, Kiang MV, et al. Implementation of the surgical safety checklist in South Carolina hospitals is associated with improvement in perceived perioperative safety. J Am Coll Surg. 2016;222(5):725-36.e5. https:// doi.org/10.1016/j.jamcollsurg.2015.12.052

19. Kain ZN, Vakharia S, Garson L, Engwall S, Schwarzkopf R, Gupta $\mathrm{R}$, et al. The perioperative surgical home as a future perioperative practice model. Anesth Analg [Internet]. 2014 [acessado em 3 maio 2019];118(5):1126-30. Disponível em: https://www.ncbi.nlm.nih.gov/ pubmed/24781578 https://doi.org/10.1213/ane.0000000000000190

20. Walters TL, Howard SK, Kou A, Bertaccini EJ, Harrison TK, Kim TE, et al. Design and implementation of a perioperative surgical home at a veterans affair hospital. Semin Cardiothorac Vascular Anesth [Internet]. 2015 [acessado em 3 maio 2019];20(2):133-40. Disponivel em: https://doi.org/10.1177\%2F1089253215607066 